\title{
FEDSM2002-31259
}

\section{ELLIPTIC RELAXATION OF A TENSOR REPRESENTATION OF THE PRESSURE-STRAIN AND DISSIPATION RATE}

\author{
John R. Carlson \\ Computational Modeling \& Simulation Branch \\ NASA Langley Research Center \\ Hampton, Virginia 23681 \\ Email: j.r.carlsonQlarc.nasa.gov
}

\author{
Thomas B. Gatski $i^{1}$ \\ Computational Modeling \& Simulation Branch \\ NASA Langley Research Center \\ Hampton, Virginia 23681 \\ Email: t.b.gatskiQlarc.nasa.gov
}

\begin{abstract}
A formulation to include the effects of wall-proximity in a second moment closure model is presented that utilizes a tensor representation for the redistribution term in the Reynolds stress equations. The wall-proximity effects are modeled through an elliptic relaxation process of the tensor expansion coefficients that properly accounts for both correlation length and time scales as the wall is approached. DNS data and Reynolds stress solutions using a full differential approach at channel Reynolds number of 590 are compared to the new model.
\end{abstract}

\section{INTRODUCTION}

The theoretical development of higher-order closure models, such as Reynolds stress models, have primarily been formulated based on high Reynolds number assumptions. Accounting for the influence of solid boundaries on these closure models has usually evolved from either a wall function approach or a modification to the high Reynolds number form of the pressure-related correlations and tensor dissipation rate, and predicated on the near-wall asymptotic behavior of the various velocity second moments (e.g. Hanjalić 1994).

A more broad based attempt to account for the proximity of a solid boundary is the elliptic relaxation approach introduced about a decade ago (Durbin, 1991), and further developed for second-moment closures (Durbin, 1993; Wizman, Laurence, Kanniche, Durbin \& Demuren, 1996; Manceau and Hanjalić, 2000, Manceau, Carlson \& Gatski, submitted for publication). In its two-equation form, the

\footnotetext{
${ }^{1}$ Address all correspondence to this author.
}

$v^{2}-f$ model has been applied to a variety of flows (e.g. Durbin, 1995; Pettersson Reif, Durbin \& Ooi, 1999). The new approach outlined here introduces a tensor function representing the combined effects of a near-wall velocitypressure gradient correlation and anisotropic dissipation rate that asymptotes to a high Reynolds number form away from solid boundaries through an elliptic relaxation equation for polynomial expansion coefficients.

With the development of a generalized methodology for constructing tensor representations for the implicit algebraic turbulent anisotropy equations (Gatski \& Jongen, 2000 ), it is possible to similarly construct tensor representations of only the redistributive part of the Reynolds stress equations (i.e. pressure-strain rate correlation and anisotropic dissipation rate equations). The elliptic relaxation formulation outlined here can be viewed as an intermediate step between a fully explicit algebraic Reynolds stress model and a full differential Reynolds stress model. 'The intermediate step being the relaxation of the expansion coefficients of the tensor representation rather than that of the redistribution function itself.

Comparisons are made with direct numerical simulation channel flow at a Reynolds number based on friction velocity of 590 . The comparisons include the mean flow, t.urbulent kinetic energy and the Reynolds stresses for solutions from both the relaxation of the redistribution terms and relaxation of the corresponding expansion coefficients. 


\section{THEORETICAL BACKGROUND AND DEVELOPMENT}

In this section, a mathematical framework is outlined that allows for the construction of a set of elliptic relaxation equations for the polynomial expansion coefficients in a tensor representation of the redistribution terms in the Reynolds stress transport equation.

The starting point in developing a elliptic relaxation formulation is the transport equation for the Reynolds stresses which can be written as

$$
\begin{aligned}
\frac{D \tau_{i j}}{D t}=-\tau_{i k} \frac{\partial \bar{U}_{j}}{\partial x_{k}} & -\tau_{j k} \frac{\partial \bar{U}_{i}}{\partial x_{k}}+\varepsilon K f_{i j}-\frac{\tau_{i j}}{K} \varepsilon \\
& +\frac{\partial}{\partial x_{l}}\left(C_{\mu} \frac{\tau_{l k}}{\sigma_{K}} \tau_{c} \frac{\partial \tau_{i j}}{\partial x_{k}}\right)+\nu \nabla^{2} \tau_{i j}
\end{aligned}
$$

where the turbulent transport term, which included expressions for the triple-moment and the pressure transport, is modeled using a gradient-diffusion model $\left(C_{\mu}=0.15\right.$, and $\tau_{c}$ is a composite time scale defined in Eq. (9)). An anisotropic dissipation rate model has been introduced (e.g. Manceau, $2000)$ into the redistributive term, $\phi_{i j}$, so that $\varepsilon K f_{i j}$ is given by

$$
\varepsilon K f_{i j}=\phi_{i j}-2 \varepsilon\left(d_{i j}-b_{i j}\right)
$$

where the Reynolds stress anisotropy $b_{i j}$ and the dissipation rate anisotropy $d_{i j}$ are

$$
b_{i j}=\frac{\tau_{i j}}{2 K}-\frac{\delta_{i j}}{3}, \quad d_{i j}=\frac{\varepsilon_{i j}}{2 \varepsilon}-\frac{\delta_{i j}}{3}
$$

The wall proximity effect is introduced through an elliptic relaxation of the redistributive model $f_{i j}$.

Solutions are obtained for the $\tau_{11}$ and $\tau_{22}$ normal Reynolds stresses, and the $\tau_{12}$ shear stress. The transport equation for the turbulent kinetic energy is used in lieu of the third normal stress $\tau_{33}$

$$
\frac{D K}{D t}=\mathcal{P}-\varepsilon+\frac{\partial}{\partial x_{l}}\left(C_{\mu} \frac{\tau_{k}}{\sigma_{K}} \tau_{c} \frac{\partial K}{\partial x_{k}}\right)+\nu \nabla^{2} K
$$

and the associated modeled transport equation for the turbulent dissipation rate, $\varepsilon$, is

$$
\frac{D \varepsilon}{D t}=\frac{C_{\varepsilon 1}^{*} \mathcal{P}-C_{\varepsilon 2} \varepsilon}{\tau_{c}}+\frac{\partial}{\partial x_{l}}\left(C_{\mu} \frac{\tau_{l k}}{\sigma_{\varepsilon}} \tau_{c} \frac{\partial \varepsilon}{\partial x_{k}}\right)+\nu \nabla^{2} \varepsilon
$$

with $\sigma_{K}=1.0, \quad \sigma_{\varepsilon}=1.3, \quad C_{\varepsilon 2}=1.83, C_{\mu}=0.15$ and

$$
C_{\varepsilon 1}^{*}=1.44\left(1+a_{1} \frac{\mathcal{P}}{\varepsilon}\right)
$$

where $a_{1}=0.09$. Note that this form of the dissipation rate equation (Durbin 1993, Manceau \& Hanjalić, 2000) has introduced a composite time scale, $\tau_{c}$, that replaces the ratio $K / \varepsilon$ in both the production and destruction of dissipation terms.

\section{Elliptic Relaxation Methodology: $\tau$ - $\mathbf{f}$ Model}

The rescaled elliptic relaxation equation is driven by the high-Reynolds number form of the pressure-strain correlation, $\Pi$, and a contribution from the Reynolds stress anisotropy, $2 \varepsilon_{c} b_{i j}$ (away from the wall the dissipation rate is assumed to be isotropic $d_{i j}=0$ )

$$
\left(1-L^{2} \nabla^{2}\right) f_{i j}=\frac{1}{\varepsilon h}\left(\Pi_{i j}^{h}+2 \varepsilon_{c} b_{i j}\right)
$$

where

$$
\varepsilon_{c}=\frac{K}{\tau_{c}}
$$

with the relaxation scales defined as

$$
\begin{gathered}
L=C_{L} \max \left[\frac{K^{3 / 2}}{\varepsilon}, C_{L_{K}}\left(\frac{\nu}{\varepsilon}\right)^{1 / 4}\right] \\
\tau=\frac{K}{\varepsilon}, \quad \tau_{c}=\max \left[\tau, C_{\tau_{K}}\left(\frac{\nu}{\varepsilon}\right)^{1 / 2}\right]
\end{gathered}
$$

where $C_{L}=0.16, C_{L_{\kappa}}=80$ and $C_{\tau_{K}}=6$ were used for the present study and were adopted from previous elliptic relaxation formulations.

In the current formulation, the goal is to implement a tensor representation for the relaxed redistribution function $f_{i j}$. As will be further discussed in the next section, such a representation would be consistent with a linear pressurestrain rate model. Previous implementations of the elliptic relaxation procedure (Manceau \& Hanjalić, 2000) using the SSG pressure strain rate model (Speziale et al., 1991) utilized the full nonlinear form. The linear form of the SSG model implemented here is given by

$$
\begin{aligned}
& \mathbf{\Pi}^{h}=-\left(C_{1}^{0} \varepsilon_{c}+C_{1}^{\mathbf{1}} \mathcal{P}\right) \mathbf{b}+K C_{2} \mathbf{S} \\
& \quad+K C_{3}\left(\mathbf{b S}+\mathbf{S} \mathbf{b}-\frac{2}{3}\{\mathbf{b} \mathbf{S}\} \mathbf{I}\right)-K C_{4}(\mathbf{b} \mathbf{W}-\mathbf{W b})
\end{aligned}
$$

with $C_{0}^{1}=1.8, C_{1}^{1}=3.4, C_{2}=0.37, C_{3}=1.25, C_{4}=0.4$. Since this linear form is used, the length scale constant $C_{L}$ 
needed to be modified from the previously used value.

In the vicinity of the wall, the redistributive term is balanced by the viscous diffusion of the Reynolds stresses resulting in the boundary conditions for the $f_{i j}$ (see Table 1). Only the 22- and 12-components of $\mathbf{f}$ have determinate solutions to the near-wall balance of the stress transport equations. Manceau et al. (submitted for publication) have used $f_{11}=f_{33}=-f_{22} / 2$ as boundary conditions on the remaining components to ensure that $\mathbf{f}$ is traceless.

Table 1. RELAXATION TENSOR BOUNDARY CONDITIONS.

\begin{tabular}{ccc}
\hline Component & Wall & Centerline \\
\hline$f_{11}$ & $-\frac{1}{2} f_{22, w}$ & symmetry \\
$f_{22}$ & $\frac{-20 v^{2}}{\varepsilon_{w}^{2} y_{(1)}^{4}}$ & symmetry \\
$f_{33}$ & $-\frac{1}{2} f_{22, w}$ & symmetry \\
$f_{12}$ & $\frac{-\left.20 \overline{u v}\right|_{(1)}}{\varepsilon_{w}^{2} y_{(1)}^{4}}$ & 0 \\
\hline
\end{tabular}

\section{Representations and Elliptic Relaxation: $\tau-\beta_{n}$ Model}

While the elliptic relaxation formulation has already been applied within a full differential Reynolds stress model, a question arises about the role tensor representations can play within the framework of the elliptic relaxation procedure.

As a first step, consider only the differential elliptic relaxation equation for the velocity-pressure gradient contribution to $f_{i j}$. This can be obtained from the integral expression (e.g. Manceau and Hanjalić, 2000)

$$
\phi_{i j}^{*}(\mathbf{x})=\frac{\phi_{i j}(\mathbf{x})}{\varepsilon(\mathbf{x}) K(\mathbf{x})}=\int_{\Omega} d^{3} \mathbf{x}^{\prime}\left[\frac{\Psi_{i j}\left(\mathbf{x}, \mathbf{x}^{\prime}\right)}{\varepsilon(\mathbf{x}) K(\mathbf{x})}\right] G_{\Omega}\left(\mathbf{x}, \mathbf{x}^{\prime}\right),
$$

where

$$
\begin{aligned}
\Psi_{i j}\left(\mathbf{x}, \mathbf{x}^{\prime}\right)= & -u_{i}(\mathbf{x}) \nabla^{2} \frac{\partial p}{\partial x_{j}}\left(\mathbf{x}^{\prime}\right)-u_{j}(\mathbf{x}) \nabla^{2} \frac{\partial p}{\partial x_{i}}\left(\mathbf{x}^{\prime}\right) \\
& +\frac{2}{3} \delta_{i j} u_{k}(\mathbf{x}) \nabla^{2} \frac{\partial p}{\partial x_{k}}\left(\mathbf{x}^{\prime}\right)
\end{aligned}
$$

and $G_{\Omega}\left(\mathbf{x}, \mathbf{x}^{\prime}\right)$ is approximated by the free-space Greens function $G_{\Omega}\left(\mathbf{x}, \mathbf{x}^{\prime}\right)=(4 \pi r)^{-1}$ with $r=\left\|\mathbf{x}^{\prime}-\mathbf{x}\right\|$. The tensor function(s) $\Psi_{i j}$ (and $\phi_{i j}$ ) can be represented by a polynomial expansion of basis tensors just as the associated Reynolds stress anisotropy tensor $b_{i j}$. For such a basis given by $T_{i j}^{(m)}(\mathbf{x})(m=1, \ldots, N)$, the representation

$$
\Psi_{i j}^{*}\left(\mathbf{x}, \mathbf{x}^{\prime}\right)=\frac{\Psi_{i j}\left(\mathbf{x}, \mathbf{x}^{\prime}\right)}{\varepsilon(\mathbf{x}) K(\mathbf{x})}=\sum_{n=1}^{\mathrm{N}} \gamma_{n}\left(\mathbf{x}, \mathbf{x}^{\prime}\right) T_{i j}^{(n)}(\mathbf{x})
$$

is assumed. A tensor scalar product (denoted by $[:]$ ) between each basis tensor and the representation given in Eq. (13) can be formed, and this leads (using matrix notation for convenience) to

$$
\begin{aligned}
{\left[\phi^{*}(\mathbf{x}): \mathbf{T}^{(m)}(\mathbf{x})\right] } & =\int_{\Omega} d^{3} \mathbf{x}^{\prime}\left[\boldsymbol{\Psi}^{*}\left(\mathbf{x}, \mathbf{x}^{\prime}\right): \mathbf{T}^{(m)}(\mathbf{x})\right] G_{\Omega}\left(\mathbf{x}, \mathbf{x}^{\prime}\right) \\
& =\int_{\Omega} d^{3} \mathbf{x}^{\prime} \gamma_{n}\left(\mathbf{x}, \mathbf{x}^{\prime}\right) \mathcal{T}_{n m} G_{\Omega}\left(\mathbf{x}, \mathbf{x}^{\prime}\right)
\end{aligned}
$$

where

$$
\mathcal{T}_{n m}=\left[\mathbf{T}^{(n)}(\mathbf{x}): \mathbf{T}^{(m)}(\mathbf{x})\right]
$$

Since the functional dependency of $\left[\mathbf{T}^{(n)}(\mathbf{x}): \mathbf{T}^{(m)}(\mathbf{x})\right]$ is only on $\mathbf{x}, \mathrm{Eq}$. (14) can be rewritten as

$$
\left[\boldsymbol{\phi}^{*}(\mathbf{x}): \mathbf{T}^{(m)}(\mathbf{x})\right] \mathcal{T}_{n m}^{-1}=\int_{\Omega} d^{3} \mathbf{x}^{\prime} \gamma_{n}\left(\mathbf{x}, \mathbf{x}^{\prime}\right) G_{\Omega}\left(\mathbf{x}, \mathbf{x}^{\prime}\right)
$$

where the scalar function $\gamma_{m}\left(\mathbf{x}, \mathbf{x}^{\prime}\right)$ is modeled as

$$
\gamma_{m}\left(\mathbf{x}, \mathbf{x}^{\prime}\right)=\gamma_{m}\left(\mathbf{x}^{\prime}, \mathbf{x}^{\prime}\right) \exp \left(-\frac{r}{L_{m}}\right)
$$

If a tensor representation of the form

$$
\boldsymbol{\phi}^{*}(\mathbf{x})=\sum_{n=1}^{\mathrm{N}} \beta_{n} \mathbf{T}^{(n)}
$$

is assumed for $\phi^{*}(\mathbf{x})$, then Eq. (16) can be rewritten as

$$
\beta_{m}(\mathbf{x})=\int_{\Omega} d^{3} \mathbf{x}^{\prime} \gamma_{m}\left(\mathbf{x}^{\prime}, \mathbf{x}^{\prime}\right) \frac{\exp \left(-r / L_{m}\right)}{4 \pi r}
$$

This leads directly to the differential formulation

$$
\left(1-L_{m}^{2} \nabla^{2}\right) \beta_{m}(\mathbf{x})=-L_{m}^{2} \gamma_{m}(\mathbf{x}, \mathbf{x})=\beta_{m}^{h}(\mathbf{x})
$$

where $\beta_{m}^{h}(\mathbf{x})$ are the expansion coefficients from the tensor representation of the quasi-homogeneous form of the 
pressure-strain rate correlation $\phi^{*}$

$$
\beta_{m}^{h}(\mathbf{x})=\frac{1}{\varepsilon K}\left[\mathbf{\Pi}^{h}(\mathbf{x}): \mathbf{T}^{(s)}(\mathbf{x})\right] \mathcal{T}_{m s}^{-1}
$$

In this study, the pressure-strain rate model used in the elliptic relaxation of the expansion coefficients differs slightly from the form used in the full differential form and given in Eq. (10)

$$
\begin{aligned}
\boldsymbol{\Pi}^{h} & =-\left(C_{1}^{0}+C_{1}^{1} \frac{\mathcal{P}}{\varepsilon}\right) \varepsilon_{c} \mathbf{b}+K C_{2} \mathbf{S} \\
& +K C_{3}\left(\mathbf{b S}+\mathbf{S} \mathbf{b}-\frac{2}{3}\{\mathbf{b S}\} \mathbf{I}\right)-K C_{4}(\mathbf{b} \mathbf{W}-\mathbf{W b}) .
\end{aligned}
$$

A comparison of Eqs. (10) and (22) shows that the (slow) term proportional to $\mathbf{b}$ has been modified so that $\varepsilon_{c}$ now affects the entire term.

One of the improvements in the current elliptic relaxation formulation is that the scaled velocity pressuregradient correlation is $\mathcal{O}(1)$ in the log-layer region. This rescaling negated the adverse influence of the elliptic operator in the log-layer in the Durbin formulation. In order to insure the same benign effect of the elliptic operator on the expansion coefficients $\beta_{m}$, it is necessary to introduce a normalized set of basis tensors.

In previous representations of the Reynolds stress anisotropy tensor, the basis tensors used in a three-term expansion were $\mathbf{S}, \mathbf{S W}-\mathbf{W S}$, and $\mathbf{S}^{2}-\left\{\mathbf{S}^{2}\right\} \mathbf{I} / 3$. In the log-layer, where the velocity gradient has a $y^{-1}$ behavior, this then requires that the corresponding expansion coefficients $\beta_{1}, \beta_{2}$, and $\beta_{3}$ to have a $y, y^{2}$, and $y^{2}$ behavior, respectively, in order to insure that $\phi^{*}$ behaved as $\mathcal{O}(1)$. Unfortunately, such a behavior would once again introduce an amplification effect by the elliptic operator on the expansion coefficients. This problem can be easily remedied by introducing a scaled basis set of the form

$$
\begin{array}{r}
\hat{\mathbf{T}}^{(1)}=\mathbf{S}^{*}, \quad \hat{\mathbf{T}}^{(2)}=\mathbf{S}^{*} \mathbf{W}^{*}-\mathbf{W}^{*} \mathbf{S}^{*} \\
\hat{\mathbf{T}}^{(3)}=\mathbf{S}^{* 2}-\frac{1}{3}\left\{\mathbf{S}^{* 2}\right\} \mathbf{I},
\end{array}
$$

where $\mathbf{S}^{*}=\mathbf{S} /\left\{\mathbf{S}^{2}\right\}^{1 / 2}$ and $\mathbf{W}^{*}=\mathbf{W} /\left\{\mathbf{S}^{2}\right\}^{1 / 2}$. This scaled now makes the behavior of both the expansion coefficients and basis tensors of $\mathcal{O}(1)$ in the log-layer, which then precludes any adverse effect of the elliptic operator in the relaxation equation given in $\mathrm{Eq}$. (20).

These results can be extended to the elliptic relaxation function $f_{i j}$. In the full differential form, the relaxation function $f_{i j}$ is composed of both the velocity-pressure gradient component as well as a contribution from the Reynolds stress and dissipation rate anisotropies (see Eq. (2)). The corresponding elliptic relaxation equation for the expansion coefficients is then given by

$$
\left(1-L_{m}^{2} \nabla^{2}\right) \beta_{m}=\frac{1}{\varepsilon K}\left(\left[\boldsymbol{\Pi}^{h}: \hat{\mathbf{T}}^{(m)}\right]+2 \varepsilon_{c}\left[\mathbf{b}: \hat{\mathbf{T}}^{(m)}\right]\right)
$$

where, for the most general formulation, each coefficient can have an associated length scale

$$
L_{m}=C_{L_{m}} \max \left[\frac{K^{-3 / 2}}{\varepsilon}, C_{L_{K_{m}}}\left(\frac{\nu}{\varepsilon}\right)^{1 / 4}\right]
$$

The $\beta_{m}$ boundary conditions are listed in Table 2 as functions of $f_{i j}$.

Table 2. $\beta_{m}$ BOUNDARY CONDITIONS.

\begin{tabular}{ccc}
\hline$\beta_{m}$ & Wall & Centerline \\
\hline$\beta_{1}$ & $\frac{-\left.20 \sqrt{2} \overline{u v}\right|_{(1)}}{\varepsilon_{w}^{2} y_{(1)}^{4}}$ & $\frac{\left[\Pi: \hat{\mathrm{T}}^{(1)}\right]^{h}}{\varepsilon K}$ \\
$\beta_{2}$ & $\frac{-\left.15 \overline{v^{2}}\right|_{(1)}}{\varepsilon_{w}^{2} y_{(1)}^{4}}$ & $\frac{\left[\Pi: \hat{\mathrm{T}}^{(2)}\right]^{h}}{\varepsilon K}$ \\
& $\frac{-\left.30 \overline{v^{2}}\right|_{(1)}}{\beta_{3}}$ & $\frac{\left[\Pi: \hat{\mathrm{T}}^{(3)}\right]^{h}}{\varepsilon K}$ \\
\hline
\end{tabular}

\section{DISCUSSION}

\section{Solution \& Calibration}

For the channel flow used in this study the equations were non-dimensionalized by the bulk viscosity and friction velocity at the wall (i.e. wall-units). The physical aspect of the channel flow was defined by the Reynolds number based on channel half-height, $R e_{\tau}$. A one-dimensional finitedifference code was used for all computations. The differencing template was node-centered with clustering close to the wall using an exponential stretching function. The 500 node grid had the first point at a height of 0.1 wall units. The channel Reynolds number determined the grid height. The $K$ and $\varepsilon$ equations were implicitly coupled as were the $\tau_{i j}-f_{i j}$ equations. The variables $U, K, \varepsilon$ and the $\tau_{i j}$ were solved in a time-dependent mode and were updated by the time step $\Delta t$. Time stepping was not used in the solution of the $f_{i j}$ equations. 


\section{Comparison of the Models}

Figures 1 to 6 show the predictive performance of both the $\tau_{i j}-f_{i j}$ and $\tau_{i j}-\beta_{n}$ formulations. As the figures show, the two methods are coincident with the only difference being in the 22-component of the pressure strain rate correlation. (In this figure the DNS data is the unaltered $\left(\tau_{c}=\tau\right)$ high Reynolds number results so that in the vicinity of the wall the data differs significantly from the computed results.) This difference is due to the slight change in the defining relation for $\Pi^{h}$ (cf. Eqs. (10) and (22)) used in the $\tau_{i j}-\beta_{n}$ formulation. Even with this slight change, it is interesting to note that the placement of the composite dissipation rate only has a localized effect on the pressurestrain rate component. There were no noticeable differences in the other computed quantities such as the mean flow, turbulent kinetic energy or Reynolds stresses due to the position of this term.

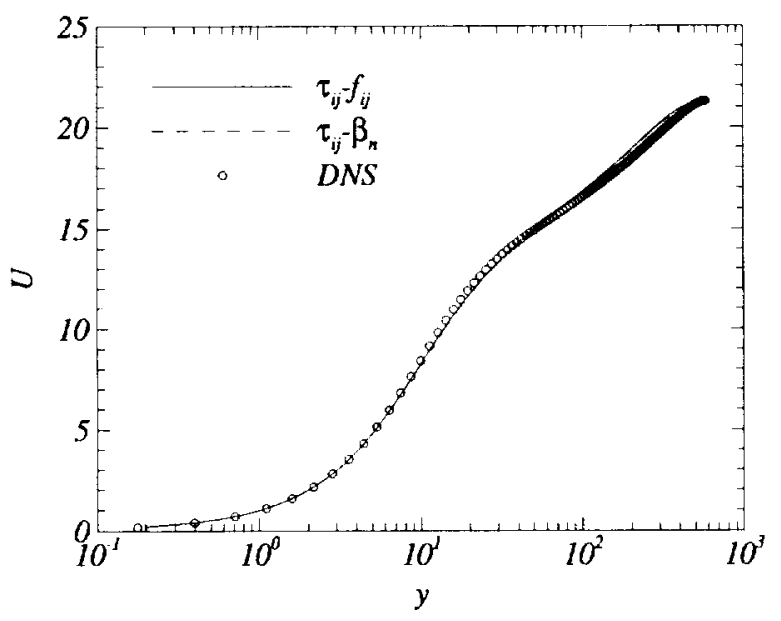

Figure 1. MEAN VELOCITY DISTRIBUTIONS.

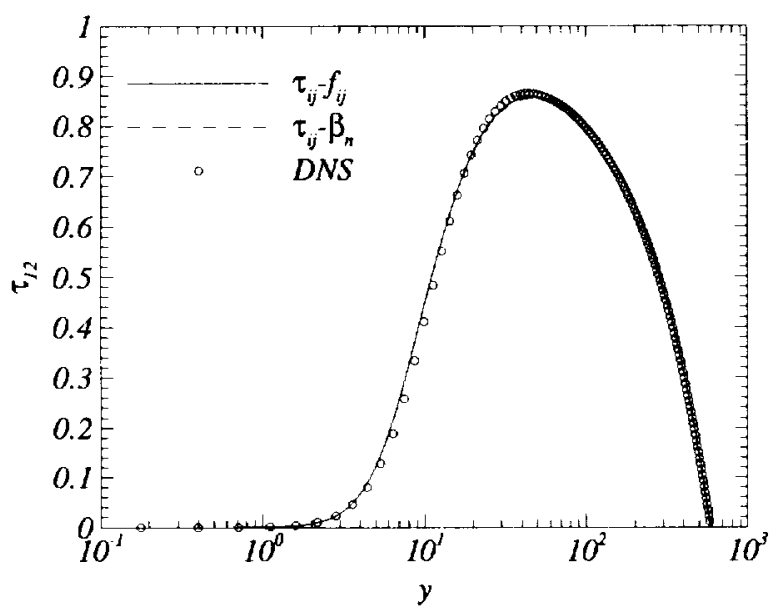

Figure 2. SHEAR STRESS DISTRIBUTIONS.

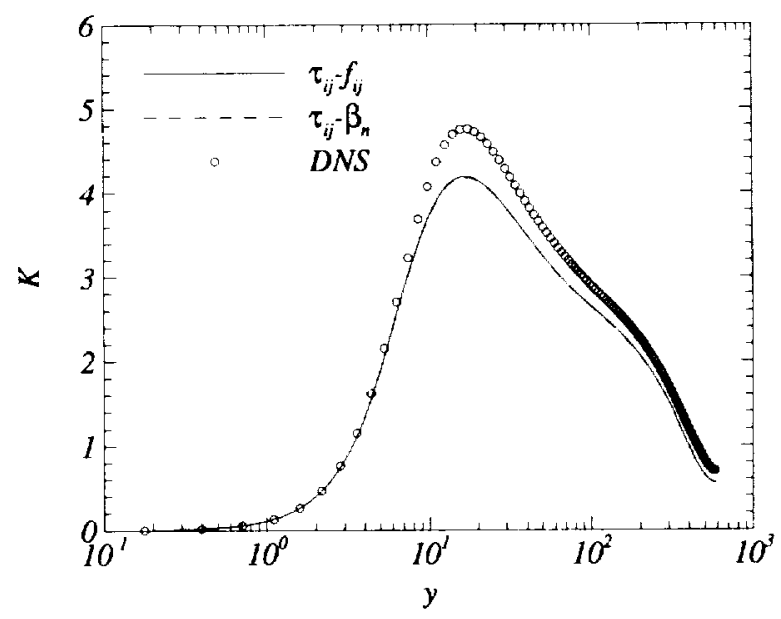

Figure 3. TURBULENT KINETIC ENERGY DISTRIBUTIONS.

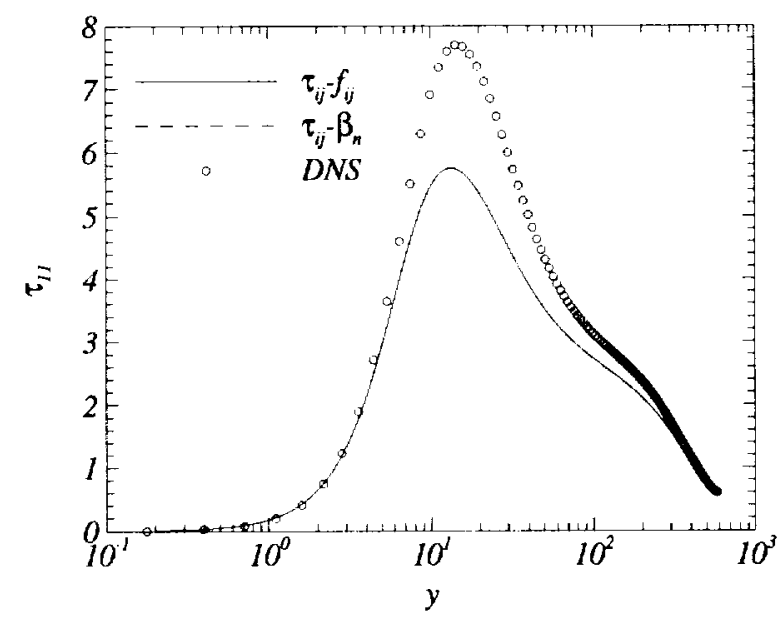

Figure 4. $\tau_{11}$ NORMAL STRESS DISTRIBUTIONS.

The consistency between the two formulations across the channel can be shown analytically. Since, for twodimensional flows, a three-term basis set is sufficient to represent a tensor function that only depends on the tensors $\mathbf{S}$ and $\mathbf{W}$. This implies that the effect of the relaxation term $\varepsilon h \mathbf{f}$ should be reproduced by a properly formulated relaxation procedure for the expansion coefficients. Consider, for example, the right hand side of the $\beta_{1}$ equation (24) expanded and expressed in terms of the invariants formed from $\mathbf{b}, \mathbf{S}$ and $\mathbf{W}$

$$
\begin{aligned}
\left(\left[\boldsymbol{\Pi}^{h}: \hat{\mathbf{T}}^{(1)}\right]+\right. & \left.2 \varepsilon_{c}\left[\mathbf{b}: \hat{\mathbf{T}}^{(1)}\right]\right)=-\left(C_{1}^{0}+C_{1}^{1} \frac{\mathcal{P}}{\varepsilon}\right) \varepsilon_{c}\left\{\mathbf{b} \mathbf{S}^{*}\right\} \\
& +K C_{2}\left\{\mathbf{S S}^{*}\right\}+2 K C_{3}\left\{\mathbf{b S S}^{*}\right\} \\
& -2 K C_{4}\left\{\mathbf{b} \mathbf{W} \mathbf{S}^{*}\right\}+2 \varepsilon_{c}\left\{\mathbf{b S}^{*}\right\} .
\end{aligned}
$$




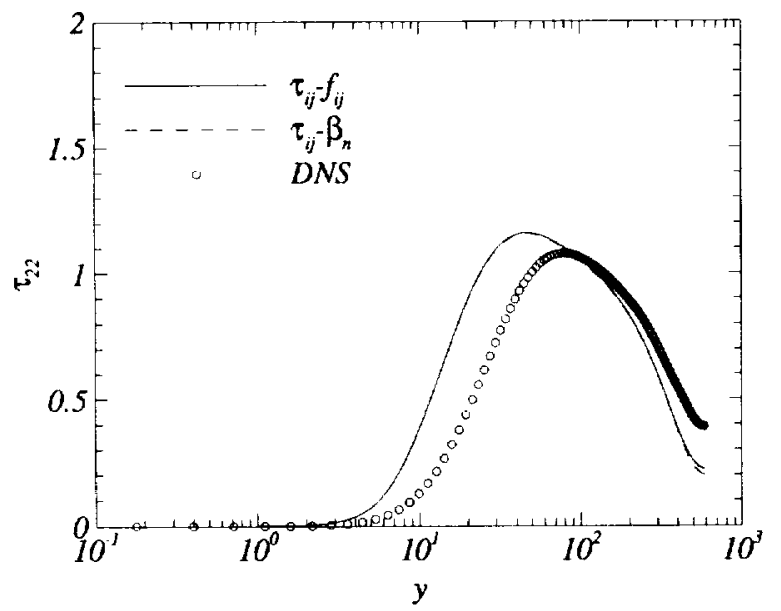

Figure 5. $\tau_{22}$ NORMAL STRESS DISTRIBUTIONS.

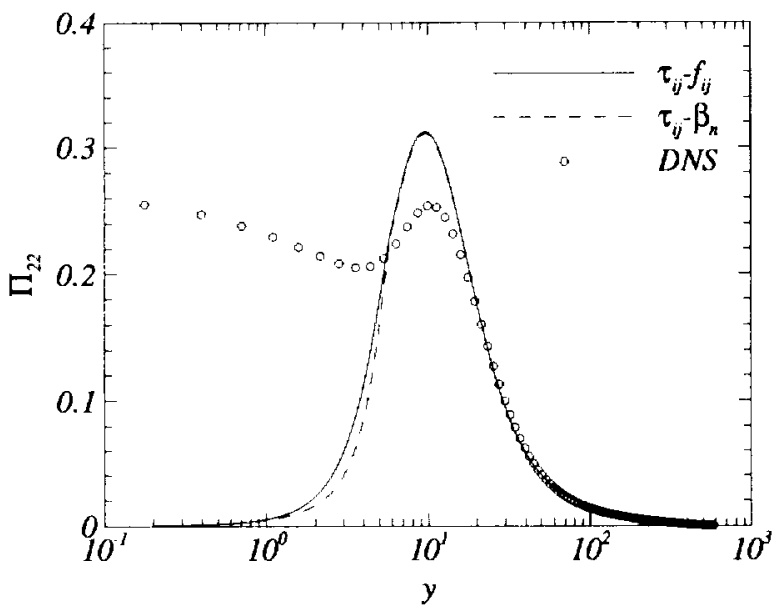

Figure 6. $\Pi_{22}$ COMPONENT OF THE PRESSURE-STRAIN RATE CORRELATION.

The only non-zero components of $\mathbf{S}$ and $\mathbf{W}$ yield $S_{12}^{*}=$ $1 / \sqrt{2}=W_{12}^{*}$. If these values are used in Eq. (26), the resulting expression is proportional to the corresponding behavior of the right hand side of Eq. (6) for the $f_{12}$ component $\left(f_{12}=\beta_{1} \hat{\mathbf{T}}^{(1)}\right)$,

$$
\left(\left[\boldsymbol{\Pi}^{h}: \hat{\mathbf{T}}^{(1)}\right]+2 \varepsilon_{c}\left[\mathbf{b}: \hat{\mathbf{T}}^{(1)}\right]\right)=\sqrt{2}\left(\Pi_{12}^{h}+2 \varepsilon_{c} b_{12}\right)
$$

Since $f_{12}=\beta_{1} / \sqrt{2}$, Eq. (27) shows that the results from the $f_{i j}$ formulation and the expansion coefficient formulation are equivalent. Similar results would be obtained for the other $\beta_{n}-f_{i j}$ sets of equations.

\section{SUMMARY}

A methodology has been developed that introduces a polynomial representation of the tensor redistribution func- which two?

tion $f_{i j}$. An elliptic relaxation equation corresponding the $f_{i j}$ relaxation equation is formulated that allows for the relaxation of the polynomial expansion coefficients. The method is demonstrated here on a fully developed channel How problem and the fwe methods are found to give similar results. This consistency is further confirmed through an analysis of the right hand sides of the corresponding elliptic relaxation equations. Ths formulation is now being used in the development of a fully explicit algebraic Reynolds stress model that incorporates an elliptic relaxation equation.

\section{ACKNOWLEDGMENT}

The authors are indebted to Dr. Remi Manceau for several useful discussions during the course of this work.

\section{REFERENCES}

Durbin, P. A. Near-wall turbulence closure without 'damping functions'. Theoret. Comput. Fluid Dyn. 3, 113,1991 .

Durbin, P. A. A Reynolds stress model for near-wall turbulence. J. Fluid Mech. 249, 465-498, 1993.

Gatski, T. B. \& Jongen, T., Nonlinear eddy viscosity and algebraic stress models for solving complex turbulent flows. Progress in Aerospace Sciences, To Appear, 2000.

Hanjalić, K., Advanced turbulence closure models: a view of current status and future prospects. Int. J. Heat and Fluid Flow 15, 178-203, 1994.

Reproducing the blocking effect of the wall in one-point turbulence models. Procecdings of the European Congress Comput. Meth. Appl. Sciences and Engineering, Barcelona, Spain, 2000.

Manceau, R., Carlson, J.R. \& Gatski, T.B., A rescaled elliptic relaxation approach: Neutralizing the effect on the log-layer. Submitted for Publication, 2001.

Manceau, R. \& Hanjalić, K., A new form of the elliptic relaxation equation to account for wall effects in RANS modeling. Phys. Fluids, 12, 2345-2351, 2000.

Moser, R. D., Kim, J. \& Mansour, N. N., DNS of Turbulent Channel Flow up to $R e_{\tau}=590$. Phys. Fluids, 11, 943945. 1999.

Pettersson Reif, B. A., Durbin, P. A. \& Ooi, A., Modeling rotational effects in eddy-viscosity closures. Int. J. Heat and Fluid Flow 20, 563-573, 1999.

Speziale, C. G., Sarkar, S., and Gatski, T. B., Modelling the pressure-strain correlation of turbulence: an invariant dynamical systems approach. J. Fluid Mech., 227, 245-272, 1991.

Wizman, V., Laurence, D., Kanniche, M., Durbin, P. \& Demuren, A., Modeling near-wall effects in second-moment closures by elliptic relaxation. Int. J. Heat and Fluid Flow 17, 255-266, 1996. 